\title{
Aleitamento materno exclusivo entre profissionais de um Programa Saúde da Família
}

\author{
Exclusive breastfeeding \\ among professionals in a Family Healthcare Program
}

Maria de Fátima Costa Caminha ${ }^{1}$

Vilneide Braga Serva ${ }^{1}$

Maria Maciel Rocha dos Anjos ${ }^{1}$

Roberta Barros de Sousa Brito ${ }^{1}$

Mônica Menezes Lins ${ }^{1}$

Malaquias Batista Filho ${ }^{1}$

${ }^{1}$ Instituto de Medicina Integral Professor Fernando Figueira. Rua dos Coelhos 300, Boa Viagem. 50070-550 Recife PE. fatimacaminha@imip.org.br
Abstract Breastfeeding is considered an important strategy for survival in infancy. Consequently, strategies have been developed mainly directed towards health professionals. The scope of the study was to identify the frequency and duration of breastfeeding among professionals of a Family Health Program in the city of Recife, Pernambuco, Brazil. It is a transversal descriptive study conducted between September and November 2006 involving 37 workers and mothers of under-five's in the Family Health Program of the IV Sanitary Health District of Recife. Of the 37 professionals, two refused to participate and were therefore excluded, meaning that 35 were effectively interviewed. The mean duration of breastfeeding was four months. Comparing the workers who breastfed exclusively with those that did not, it was found that mothers who breastfed exclusively for $\geq 4$ months, had no difficulties with breastfeeding $(p=0.027)$. A tendency of statistical significance was found in relation to the use of pacifiers among children whose mothers did not breastfeed exclusively ( $p=0.051)$. The difficulties found during breastfeeding among the workers of the Family Health Program of the IV Sanitary Health District and the use of pacifiers among their children are problems that deserve attention, due to the fact that they represent a group that serves as a model for the community.

Key words Breastfeeding, Mother's milk, Family Healthcare Program
Resumo A amamentação é uma estratégia importante de sobrevivência infantil. Assim, vêm sendo desenvolvidas estratégias de sensibilização, especialmente direcionadas aos profissionais de saúde. O estudo objetivou identificar a frequência e o período de aleitamento materno exclusivo entre profissionais de um Programa Saúde da Família da cidade do Recife, Pernambuco, Brasil. Trata-se de um estudo transversal descritivo, envolvendo 37 mulheres, mães de crianças $<5$ anos, funcionárias do Programa Saúde da Família, do Distrito Sanitário IV do Recife, entre setembro e novembro de 2006. De 37 profissionais, duas foram excluidas por recusa, resultando em 35 efetivamente entrevistadas. A mediana do aleitamento materno exclusivo foi de quatro meses. Comparando-se as profissionais que amamentaram exclusivamente ou não, foi encontrado que as mulheres que amamentaram exclusivamente por $\geq 4$ meses não tiveram dificuldades para amamentar ( $p=0,027)$. Foi encontrada uma tendência à significância quanto ao uso de chupeta em crianças no grupo de mulheres que não amamentaram exclusivamente ( $p=0,051)$. Conclui-se que as dificuldades encontradas na amamentação entre as profissionais de Programa Saúde da Família estudadas e o uso de chupeta por seus filhos merecem destaque, por se tratar de um grupo que serve de modelo para a população.

Palavras-chave Aleitamento materno, Leite materno, Programa Saúde da Família 


\section{Introdução}

A amamentação é considerada uma estratégia importante de sobrevivência infantil pelo Fundo das Nações Unidas para a Infância (Unicef), pela Organização Mundial da Saúde (OMS) e por órgãos de proteção à criança. A proteção conferida pelo leite humano contra infecções comuns em crianças repercutiu na redução da mortalidade infantil. Cerca de $13 \%$ a $15 \%$ de todas as mortes de crianças abaixo de cinco anos em todo o mundo, sendo $50 \%$ por doenças respiratórias e $66 \%$ por diarreia, poderiam ser prevenidas com o aleitamento materno ${ }^{1}$.

No Brasil, até o início de 1980, as atividades de incentivo ao aleitamento materno ocorriam de forma isolada e envolviam, sobretudo, o setor da saúde. Em janeiro de 1981, o Ministério da Saúde (MS) adotou as recomendações formuladas na reunião de Genebra em 1979 e, com o suporte do Unicef, lançou o Programa Nacional de Incentivo ao Aleitamento Materno (PNIAM), no Instituto Nacional de Alimentação e Nutrição².

O MS utilizou como estratégia de sensibilização ao aleitamento materno a formação de grupos interministeriais e interdisciplinares de trabalho sobre o tema, apoio a pesquisas, veiculação de literatura científica, capacitação de recursos humanos, implantação de alojamento conjunto com revisão das rotinas dos serviços de saúde, desenvolvimento e consolidação da rede nacional de bancos de leite humano, entre outras ações².

Todo esse empenho é devido às numerosas vantagens do aleitamento materno tanto para o binômio mãe-filho como para a sociedade. Além de suprir todas as necessidades nutritivas da criança nos primeiros seis meses de vida, o leite materno proporciona um adequado desenvolvimento biopsicossocial, protege contra infecções, alergias, problemas odontológicos e fonoaudiólogos, favorece o vínculo afetivo entre mãe e filho, acelera a involução uterina, diminui o risco de câncer de mama, ajuda a retardar nova gestação e, para a família, representa economia financeira ${ }^{3}$.

Devido a esta reconhecida importância, a amamentação passou a ser garantida por lei no artigo $9^{\circ}$ do Estatuto da Criança e do Adolescente ${ }^{4}$. Entretanto, o resultado do último inquérito nacional, realizado nas capitais brasileiras e no Distrito Federal, encontrou uma duração mediana de aleitamento materno de dez meses, sendo de apenas 23 dias a duração da amamentação exclusiva ${ }^{1}$.

$\mathrm{O}$ aleitamento materno exclusivo, do nascimento até os seis meses de vida, é considerado a forma ideal de alimentação para o bebế $\hat{e}^{5}$ De acor- do com Contrim et al. ${ }^{6}$ e Lamonier ${ }^{7}$, o grande desafio está em reduzir a introdução de bicos artificiais nesse período como mamadeiras, chupetas e mordedores, que se caracterizam como parte da nossa cultura social e podem induzir ao desmame precoce e levar a alteração nas habilidades motoras orais do recém-nascido e lactente, as quais estão intimamente relacionadas à alimentação. O desenvolvimento da tonicidade orofacial é adequado quando o lactente mantém uma amamentação exclusiva por pelo menos seis meses, levando a um bom mecanismo de sucção, erupção correta dos dentes e adequada oclusão, mastigação efetiva, articulação correta dos sons, entre outros aspectos.

A amamentação é um processo natural. Entretanto, é comum deparar-se com mães em dificuldades durante esse processo, o que pode desencadear o desmame precoce. Diante das dificuldades maternas durante o início da amamentação, o profissional de saúde, além de conhecimento teórico e competências clínicas em aleitamento materno, necessita de habilidades de comunicação ${ }^{1}$. Para adquirir essa habilidade, é importante diferenciar entre o simples ato de aconselhar e o aconselhamento. Se aconselhar é dizer o que se deve fazer, aconselhamento é uma forma de atuação em que o profissional escuta e compreende e, desta forma, oferece ajuda para que a mãe planeje, tome decisões e se fortaleça para lidar com pressões, aumentando sua autoestima e autoconfiançå ${ }^{8}$.

Estudo realizado em São Paulo, avaliando sensibilização, conhecimentos e capacitação das Equipes de Saúde da Família (ESF), concluiu que os profissionais entrevistados estão sensibilizados quanto à importância do aleitamento e têm conhecimento teórico sobre o tema, mas apresentam dificuldades para solucionar questões práticas sobre o manejo da amamentação, o que pode interferir de forma negativa no trabalho desenvolvido de apoio às mães lactantes ${ }^{9}$. Outro estudo de revisão de literatura, avaliando o aconselhamento em amamentação, concluiu que a prática e o conhecimento adequado dos profissionais de saúde constituem um importante instrumento para o aumento da prevalência e da duração da amamentação ${ }^{8}$.

$\mathrm{O}$ aconselhamento em amamentação deve ser iniciado ainda no pré-natal, devendo permear todo o período de lactação, para que haja continuidade do aleitamento materno, contribuindo, assim, para a saúde do binômio mãe-filho ${ }^{3}$.

$\mathrm{O}$ apoio nesse processo contribui para que a amamentação ocorra livre de dificuldades. No 
entanto, exige uma ação intersetorial entre o serviço de saúde e a família. Essa ação conjunta engloba informações sobre a amamentação e seus benefícios e o apoio emocional para uma experiência bem-sucedida ${ }^{3}$.

Devido à relevância do aleitamento materno, considerou-se importante conhecer a frequência dessa prática entre os profissionais do Programa Saúde da Família (PSF) da cidade do Recife, uma vez que são eles os responsáveis pelo aconselhamento junto à comunidade. Dessa forma, esperamos contribuir para o planejamento das ações de sensibilização da amamentação, no sentido de promover melhor qualidade de vida para a mulher, a criança e sua família.

\section{Métodos}

Realizou-se um estudo transversal envolvendo mães de crianças $<5$ anos, que trabalhavam no PSF do Distrito Sanitário IV na cidade do Recife, o qual possui 329 funcionários do PSF. Destes, apenas vinte são do sexo masculino, estando distribuídos nas seguintes categorias: 35 médicos, 35 enfermeiras, 36 auxiliares de enfermagem, 18 dentistas, 19 auxiliares de consultório dentário e 186 agentes comunitários de saúde. Foram excluídas as mães de crianças maiores de 59 meses $^{10}$.

Foi aplicado um formulário baseado na Pesquisa de Prevalência do Aleitamento Materno nas Capitais Brasileiras, estudo desenvolvido pelo MS em $2001^{2}$.

Para efeito desta pesquisa, foram consideradas em "Aleitamento Materno Exclusivo": crianças alimentadas somente com o leite materno e/ ou vitaminas e medicamentos prescritos por médicos ${ }^{11}$; "Aleitamento Materno Predominante": crianças alimentadas com leite materno às quais são administrados, adicionalmente, água, chás e sucos ${ }^{11}$; "Aleitamento": crianças que, além de mamarem ao peito, recebem alimentos artificiais ${ }^{11}$.

Para construção do banco de dados, utilizou-se programa software EPI-INFO versão 6.0 (WHO/CDC, Atlanta, GE, USA). A análise comparativa dos resultados foi realizada pelo Teste Exato de Fisher. Utilizou-se o nível de significância de 5\%. A duração do aleitamento materno exclusivo foi estimada pela mediana (intervalo interquartílico). Os dados foram processados com o software SPSS para Windows, versão 13.1 (SPSS Inc., Chicago, IL, USA). O projeto de pesquisa foi aprovado pelo Comitê de Ética em Pesquisa em Seres Humanos do Instituto MaternoInfantil Professor Fernando Figueira (IMIP).

\section{Resultados}

Das 37 profissionais do PSF inicialmente selecionadas, duas foram excluídas por recusa, resultando em 35 efetivamente entrevistadas.

A mediana do aleitamento materno exclusivo foi de quatro meses ( $1^{\circ}$ quartil $=4$ meses; e $3^{\circ}$ quartil $=6$ meses), e apenas 11 das 35 profissionais referiram o aleitamento materno exclusivo após o $4^{\circ}$ mês de lactação.

A idade das crianças variou de 12 a 59 meses, com mediana de 38 meses ( $1^{\circ}$ quartil $=23$ meses; $3^{\circ}$ quartil $=48$ meses , e a idade das profissionais variou de 24 a 38 anos, com mediana de 29 anos ( $1^{\circ}$ quartil $=28$ anos; $3^{\circ}$ quartil $=34$ anos $)$.

Mais da metade das profissionais $(60 \%)$ trabalhava como agente comunitária de saúde (ACS), eram casadas e com filho do sexo masculino. $74,3 \%$ tinham o segundo grau completo e $65,6 \%$ tinham apenas um filho (Tabela 1).

Tabela 1. Distribuição das profissionais do Programa Saúde da Família do Distrito Sanitário IV, segundo variáveis indicadoras da sua situação sociodemográfica. Recife, setembro a novembro de 2006 .

\begin{tabular}{lrr}
\hline \multicolumn{1}{c}{ Variáveis } & n & $\%$ \\
\hline Cargo/função & & \\
$\quad$ Médico & 2 & 5,7 \\
Enfermeiro(a) & 4 & 11,4 \\
Dentista & 1 & 2,9 \\
Técnico em enfermagem & 2 & 5,7 \\
ACS & 21 & 60,0 \\
ACD & 5 & 14,3 \\
Situação conjugal & & \\
Solteira & 8 & 22,9 \\
Casada no civil & 21 & 60,0 \\
Outro & 6 & 17,1 \\
Escolaridade & & \\
1 grau & 2 & 5,7 \\
20 grau & 26 & 74,3 \\
Superior & 7 & 20,0 \\
Número de filhos & & \\
1 filho(a) & 23 & 65,6 \\
2 filhos(as) & 8 & 22,9 \\
3 filhos(as) & 1 & 2,9 \\
4 filhos(as) & 3 & 8,6 \\
Sexo do filho & & \\
Masculino & 21 & 60,0 \\
Feminino & 14 & 40,0 \\
\hline
\end{tabular}

Fonte: Pesquisa IMIP.

ACS = Agente comunitária de saúde; $\mathrm{ACD}=$ Auxiliar de consultório dentário. 
Todas as entrevistadas fizeram o acompanhamento pré-natal, sendo que 76,6\% delas realizaram oito ou mais consultas. $91,3 \%$ iniciaram esse atendimento no primeiro trimestre, e destas, $42,8 \%$ no primeiro mês de gestação. Quanto à participação em palestra sobre amamentação, $40 \%$ não realizaram esse tipo de atividade. Mais da metade $(57,1 \%)$ teve parto cesariano e permaneceu ao lado do filho desde o nascimento até a alta hospitalar (62,9\%) (Tabela 2).

$\mathrm{O}$ uso de chupeta e mamadeira foi referido em $38 \%$ e $64,7 \%$, respectivamente. A grande maioria das mães $(94,1 \%)$ retornou ao trabalho aos quatro meses ou mais de vida do bebê. $61,8 \%$ do total da amostra apresentaram alguma dificuldade durante o processo de amamentação (Tabela 3 ).

Em relação às comparações de frequências das profissionais que amamentaram exclusivamente ou não, foi encontrado que as mães que amamentaram exclusivamente por $\geq 4$ meses não tiveram dificuldades para amamentar, sendo este resultado estatisticamente significante $(\mathrm{p}=0,027)$, o que não ocorreu quando relacionado ao uso

Tabela 2. Distribuição das profissionais do Programa Saúde da Família do Distrito Sanitário IV, segundo variáveis indicadoras da assistência à saúde no período da gestação. Recife, setembro a novembro de 2006.

\begin{tabular}{lrr}
\hline \multicolumn{1}{c}{ Variáveis } & n & \multicolumn{1}{c}{$\%$} \\
\hline Fez pré-natal & 35 & 100,0 \\
$\quad$ Sim & 0 & 0,0 \\
$\quad$ Não & & \\
No de consultas de pré-natal & 1 & 2,9 \\
5 consultas & 1 & 2,9 \\
6 consultas & 6 & 17,6 \\
7 consultas & 26 & 76,6 \\
8 ou mais consultas & & \\
Início do pré-natal & 32 & 91,3 \\
$\quad 1^{\circ}$ trimestre & 2 & 5,8 \\
20 trimestre & 1 & 2,9 \\
$\quad$ Não lembra & & \\
Participação em palestras & 21 & 60,0 \\
Sim & 14 & 40,0 \\
$\quad$ Não & & \\
Tipo de parto & 15 & 42,9 \\
$\quad$ Normal hospitalar & 20 & 57,1 \\
Cesário & & \\
Permanência com o filho até a alta & & 62,9 \\
Sim & 22 & 37,1 \\
Não & 13 & \\
\hline
\end{tabular}

Fonte: Pesquisa IMIP. de chupeta $(\mathrm{p}=0,051)$ e na interferência do retorno ao trabalho na amamentação $(\mathrm{p}=0,710)$ (Tabela 4).

Tabela 3. Distribuição das profissionais do Programa de Saúde da Família do Distrito Sanitário IV, segundo variáveis indicadoras de fatores motivadores e desmotivadores da amamentação. Recife, setembro a novembro de 2006.

\begin{tabular}{lrr}
\hline \multicolumn{1}{c}{ Variáveis } & n & \multicolumn{1}{c}{$\%$} \\
\hline Usa ou usou chupeta & & \\
$\quad$ Sim & 13 & 38,2 \\
$\quad$ Não & 21 & 61,8 \\
Quantos meses após o nascimento & & \\
do filho a mãe retornou ao trabalho & & \\
$\quad$ Menos de 4 meses & 2 & 5,9 \\
$\quad$ 4 meses ou mais & 32 & 94,1 \\
Dificuldade para amamentar & & \\
$\quad$ Sim & 12 & 35,3 \\
$\quad$ Não & 21 & 61,8 \\
$\quad$ Não aplicável & 1 & 2,9
\end{tabular}

Fonte: Pesquisa IMIP.

Tabela 4. Comparação das profissionais do Programa de Saúde da Família do Distrito Sanitário IV que amamentaram exclusivamente com as que não amamentaram por $\geq 4$ meses, relacionando-se dificuldade para amamentar, uso de chupeta e interferência do retorno ao trabalho na amamentação. Recife, setembro a novembro de 2006.

\begin{tabular}{|c|c|c|c|c|c|}
\hline \multirow{3}{*}{ Variáveis } & \multicolumn{4}{|c|}{$\begin{array}{c}\text { Aleitamento materno } \\
\text { exclusivo }\end{array}$} & \multirow[b]{3}{*}{ p-valor } \\
\hline & \multicolumn{2}{|c|}{ Sim } & \multicolumn{2}{|c|}{ Não } & \\
\hline & $\mathbf{n}$ & $\%$ & $\mathbf{n}$ & $\%$ & \\
\hline \multicolumn{6}{|c|}{$\begin{array}{l}\text { Dificuldade para } \\
\text { amamentar? }\end{array}$} \\
\hline Sim & 1 & 9,1 & 11 & 50,0 & \multirow{2}{*}{$0,027^{\star}$} \\
\hline Não & 10 & 90,9 & 11 & 50,0 & \\
\hline \multicolumn{6}{|l|}{ Uso de chupeta } \\
\hline Sim & 1 & 10,0 & 12 & 50,0 & \multirow{2}{*}{$0,051^{\star}$} \\
\hline Não & 9 & 90,0 & 12 & 50,0 & \\
\hline \multicolumn{6}{|c|}{$\begin{array}{l}\text { Interferência do } \\
\text { retorno ao trabalho } \\
\text { na amamentação }\end{array}$} \\
\hline Sim & 7 & 70,0 & 14 & 60,9 & \multirow{2}{*}{$0,710^{*}$} \\
\hline Não & 3 & 30,0 & 9 & 39,1 & \\
\hline
\end{tabular}

Fonte: Pesquisa IMIP.

* Teste Exato de Fisher. 


\section{Discussão}

A mediana do aleitamento materno exclusivo por quatro meses, observada nas profissionais estudadas, vem mostrar uma realidade que não é diferente da maioria das mães em nosso país, no que diz respeito ao tempo de retorno ao traba1 ho ${ }^{12,13}$. No estudo atual, $94,1 \%$ das mulheres retornaram ao trabalho ao fim da licença-maternidade (quatro meses). Embora mereça destaque que a licença-maternidade seja útil à manutenção do aleitamento materno exclusivo, outras medidas também se fazem necessárias, uma vez que a falta de condições ambientais para ordenha do leite e a ausência de creches para manter a proximidade com a criança foram as principais dificuldades apontadas pelas mulheres para a manutenção da lactação. Resultados similares também foram encontrados em outras pesquisas, como nos estudos de Rea et al. ${ }^{12}$ e Silva e Utiyama ${ }^{13}$, corroborando a Pesquisa de Prevalência do Aleitamento Materno nas Capitais Brasileiras ${ }^{2}$, em que foi postulado que o fato de a mãe não trabalhar favorecia o aleitamento materno exclusivo.

A mediana de quatro meses (160 dias), entretanto, foi muito superior à encontrada na Pesquisa Nacional $(23 \text { dias })^{2}$. Pode ter contribuído para tal resultado o fato de a Pesquisa de Prevalência do Aleitamento Materno nas Capitais Brasileiras ter sido desenvolvida com a população em geral, e a pesquisa em questão ter se limitado a um grupo de profissionais de saúde continuamente capacitados para ações de saúde relacionadas à promoção, proteção e apoio ao aleitamento materno exclusivo.

$\mathrm{Na}$ nossa casuística, da mesma forma que na Pesquisa de Prevalência do Aleitamento Materno nas Capitais Brasileiras ${ }^{2}$, o uso de bicos artificiais, entre eles a chupeta, foi uma prática frequente que mostrou uma tendência a significância estatística em relação ao desmame precoce $(\mathrm{p}=0,051)$. Provavelmente, se o tamanho amostral fosse maior, haveria de fato um resultado estatisticamente significante semelhante à Pesquisa de Prevalência do Aleitamento Materno nas Capitais Brasileiras². A presença de hábitos orais afeta o sucesso do aleitamento materno, podendo trazer, como consequência, o desmame precoce, ou seja, a criança acaba adquirindo hábitos de sucção não nutritiva, dentre eles o uso da chupeta, decorrendo em alteração no desenvolvimento motor-oral, craniofacial, no crescimento ósseo, má oclusão dentária ${ }^{14}$ e principalmente o desmame precoce ${ }^{6}$. No estudo de Soares et al. ${ }^{15}$, foi confirmado que a prática do uso de chupeta é muito arraigada na nossa cultura, mesmo em população orientada para evitá-lo, e que ocorre uma associação entre uso de chupeta e menor duração do aleitamento materno e do aleitamento materno exclusivo.

No grupo estudado, a associação significativa entre a amamentação exclusiva por quatro meses e a ausência de dificuldade para amamentar vem demonstrar estreita relação entre desmame precoce e esta última variável. Achados similares foram descritos por Ramos e Almeida ${ }^{16}$, no que diz respeito a esta associação. Uma explicação para esses resultados poderia ser a de que, no contexto estudado, profissionais - mesmo da área de saúde - podem apresentar dificuldade para amamentar, e que essas dificuldades sejam subestimadas perante a sua qualificação. Vale salientar o contraponto relacionado ao fato de os sujeitos do estudo serem profissionais de saúde, pois se de um lado a mediana do aleitamento materno exclusivo, embora não tenha sido a ideal (=6 meses), foi acima da encontrada na população em geral, dificuldades para amamentar levaram a reduzir o aleitamento materno exclusivo.

Nesse cenário das dificuldades na amamentação, o aconselhamento do profissional de saúde é de fundamental importância na ajuda à superação dessas dificuldades estabelecidas. Ele deve ocorrer em diferentes momentos: no pré-natal, na sala de parto, no alojamento conjunto e no puerpério $^{8}$. Essas informações e orientações devem se estender também à rede de apoio familiar, pois uma mãe que amamenta facilmente perde a confiança em si mesma e pode se tornar suscetível à pressão de familiares e conhecidos ${ }^{8}$. Ainda que a mãe seja uma profissional de saúde, também está sujeita às mesmas pressões familiares, sociais e emocionais, por isso se faz necessário intervir da mesma forma que as demais, pois o aconselhamento profissional vem para reforçar a autoestima e confiança na capacidade de amamentar ${ }^{8}$.

Com a obtenção do diagnóstico rápido da situação da amamentação entre os profissionais de saúde do PSF pertencentes ao Distrito Sanitário IV, esperamos contribuir para o planejamento e o redirecionamento das políticas setoriais na área de saúde da mulher e da criança, melhorando a prática do aleitamento materno exclusivo não só entre os profissionais, mas também na população assistida, uma vez que esse profissional exitoso no aleitamento materno exclusivo exemplificaria o modelo a essa prática. 


\section{Colaboradores}

MFC Caminha e VB Serva participaram da concepção, do delineamento, análise, interpretação dos dados, redação do artigo e revisão crítica, assim como foram as orientadoras deste trabalho; MMR Anjos e RBS Brito, da concepção, do delineamento, análise, interpretação dos dados e redação; MM Lins e M Batista Filho, da análise e interpretação dos dados e da revisão crítica do artigo.

\section{Referências}

1. Duncan BB, Schmidt MI, Giugliani ERJ. Medicina ambulatorial: condutas de Atenção Primária baseada em evidências. $3^{\text {a }}$ ed. Porto Alegre: Artmed; 2004.

2. Brasil. Ministério da Saúde. Pesquisa de prevalência do aleitamento materno nas capitais e no Distrito Federal: relatório. Brasília: Ministério da Saúde; 2001.

3. King FS. Como ajudar as mães a amamentar. $4^{\mathrm{a}}$ ed. Brasília: Ministério da Saúde; 2001. [documento na Internet] [acessado $2008 \mathrm{fev}$ 9]. Disponível em: http://bvsms.saude.gov.br/bvs/publicacoes/cd03_ 13.pdf

4. Brasil. Estatuto da criança e do adolescente (1990): Lei $n^{\circ} 8.069$, de 13 de julho de 1990, Lei no 8.242, de 12

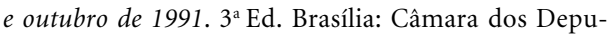
tados, Coordenação de Publicações; 2001. [documento na Internet] [acessado 2008 fev 9]. Disponível em: http://www2.camara.gov.br/publicacoes/ internet/publicacoes/estatutocrianca.pdf

5. Brasil. Ministério da Saúde. Manual de promoção do aleitamento materno: normas técnicas. Brasília: Ministério da Saúde; 1997.

6. Cotrim LC, Venâncio SI, Escuder MML. Uso de chupeta e amamentação em crianças menores de 4 meses no Estado de São Paulo. Rev Bras Saude Mater Infant [periódico na Internet]. 2002 [acessado 2008 fev 9]; 2(3):245-252. Disponível em: http:// www.scielo.br/scielo.php?script=sci_arttext \& pid $=$ S1519-38292002000300005

7. Lamounier JL. O efeito de bicos e chupetas no aleitamento materno. J Pediatr [periódico na Internet]. 2003 [acessado $2008 \mathrm{fev}$ 9]; 79(4):284-286. Disponível em: http://www.scielo.br/pdf/jped/ v79n4/v79n4a04.pdf

8. Bueno LGS, Teruya KM. Aconselhamento em amamentação e sua prática. $J$ Pediatr [periódico na Internet]. 2004 [acessado $2008 \mathrm{fev}$ 9]; 80(Supl.5):S126S130. Disponível em: http://www.scielo.br/pdf/ jped/v80n5s0/v80n5s0a03.pdf

9. Ciconi RCV, Venâncio SI, Escuder MML. Avaliação dos conhecimentos de equipes do Programa de Saúde da Família sobre o manejo do aleitamento materno em um município da região metropolitana de São Paulo. Rev Bras Saude Mater Infant [periódico na Internet]. 2004 [acessado 2008 fev 9]; 4(2):193202. Disponível em: http://www.scielo.br/scielo.php? pid $=$ S1519-38292004000200010\&script $=$ sci_arttext
10. Ford K, Labbok M. Who is breast-feeding? Implications of associated social and biomedical variables for research on the consequences of method of infant feeding. Am J Clin Nutr [ periódico na Internet]. 1990 [acessado $2008 \mathrm{fev}$ 9]; 52(3):451-456. Disponível em: http://www.ajcn.org/cgi/reprint/52/3/451

11. United Nations Children's Fund (Unicef), World Health Organization (WHO). Breastfeeding management and promotion in a baby-friendly hospital: an 18 hour course for maternity staff. New York: Unicef; 1993.

12. Rea MF, Venâncio SI, Batista LE, Santos RGS, Greiner T. Possibilidades e limitações da amamentação entre mulheres trabalhadoras formais. Rev Saude Publica [periódico na Internet]. 1997 [acessado 2008 fev 9]; 31(2):149-156. Disponível em: http://www. scielo.br/scielo.php?pid=S0034-89101997000200008\& script=sci_arttext

13. Silva IA, Utiyama SK. Situação de amamentação entre mulheres trabalhadoras e alunas de graduação de uma universidade pública. Acta Scient Cienc Saúde 2003; 25(2):215-225.

14. Ogaard B, Larsson E, Lindsten R. The effect of sucking habits, cohort, sex, intercanine arch widths, and breast or bottle feeding on posterior crossbite in Norwegian and Swedish 3-year-old children. Am J Orthod Dentofacial Orthop 1994; 106(2):161-166.

15. Soares MEM, Giugliani ERJ, Braun ML, Salgado ACN, Oliveira AP, Aguiar PR. Uso de chupeta e sua relação com o desmame precoce em população de crianças nascidas em Hospital Amigo da Criança. $J$ Pediatr [periódico na Internet]. 2003 [acessado 2008 fev 9]; 79(4):309-316. Disponível em: http://www. scielo.br/pdf/jped/v79n4/v79n4a08.pdf

16. Ramos CV, Almeida JAG. Alegações maternas para o desmame: estudo qualitativo. J Pediatr [periódico na Internet]. 2003 [acessado 2008 fev 9]; 79(5):385390. Disponível em: http://www.scielo.br/pdf/jped/ v79n5/v79n5a04.pdf

Artigo apresentado em 13/02/2008

Aprovado em 29/10/2008

Versão final apresentada em 30/11/2008 